\title{
Identification of microRNA-363-3p as an essential regulator of chondrocyte apoptosis in osteoarthritis by targeting NRF1 through the p53-signaling pathway
}

\author{
MIAO ZHANG ${ }^{1,2}$, ZHIQIANG WANG ${ }^{3},{\text { BAOJIE } \mathrm{LI}^{3}, \text { FENGYI SUN }}^{4}$, ANZHONG CHEN $^{5}$ and MINGZHI GONG ${ }^{1}$ \\ ${ }^{1}$ Department of Trauma and Orthopedics, The Second Hospital of Shandong University, Jinan, Shandong 250033; \\ Departments of ${ }^{2}$ Joint Surgery, ${ }^{3}$ Traumatic Orthopedics and ${ }^{4}$ Gynaecology, Weifang People's Hospital, Weifang, \\ Shandong 261000; ${ }^{5}$ Department of Rehabilitation, The Second People's Hospital of Liaocheng, \\ Linqing, Shandong 252600, P.R. China
}

Received April 16, 2019; Accepted November 6, 2019

DOI: $10.3892 / \mathrm{mmr} .2020 .10940$

\begin{abstract}
Osteoarthritis (OA) is a degenerative joint disease that affects the physical, and mental health of middle-aged and elderly people. The aims of the present study were to determine the biological function and molecular mechanisms of miR-363-3p in chondrocyte apoptosis. Exploration of the molecular mechanisms of OA may be helpful in the understand of the causes, and facilitating the prevention and treatment of OA. In the present study, the expression of nuclear respiratory factor1 (NRF1) was downregulated in the articular cartilage of OA rats in vivo and lipopolysaccharide (LPS)-treated chondrocytes in vitro. MicroRNAs (miRNA) are regulators of gene expression in the progression of OA. TargetScan software was used to predict that NRF1 was a potential target for miRNA (miR)-363, and this was confirmed in subsequent experiments. The expression of miR-363-3p was negatively correlated with the expression of NRF1, and its expression was significantly upregulated in OA model rats and in LPS-induced chondrocytes compared with the expression in the respective controls. In addition, the overexpression of miR-363-3p increased the levels of interleukin (IL)-1 $\beta$, IL-6 and tumor necrosis factor- $\alpha$ in vivo, and was demonstrated to promote chondrocyte injury and apoptosis by Safranin O staining and TUNEL. Moreover, the inhibition of miR-363-3p expression increased the expression of NRF1 and protected chondrocytes from apoptosis in vitro and in vivo, whereas the overexpression of miR-363-3p downregulated NRF1 expression and promoted LPS-induced chondrocyte apoptosis through the p53 pathway in vitro. The results of this study suggested that miR-363-3p-mediated
\end{abstract}

Correspondence to: Dr Mingzhi Gong, Department of Trauma and Orthopedics, The Second Hospital of Shandong University, 247 Beiyuan Street, Jinan, Shandong 250033, P.R. China

E-mail: doc_gmz@163.com

Key words: osteoarthritis, chondrocytes, apoptosis, microRNA-363, p53, nuclear respiratory factor 1 inhibition of NRF1may be associated with chondrocyte apoptosis in OA.

\section{Introduction}

Osteoarthritis (OA) is a degenerative condition of the joints that significantly affects the physical and mental health of middle-aged and elderly people (1). There are $>360$ million people suffering from OA all over the world, and the treatment of OA is an economic burden on society (2). The main pathological feature is the degeneration of articular cartilage, which clinically manifests as frequently recurring joint pain, joint dysfunction and other symptoms, such as joint swelling, stiffness and deformity (3). The main function of articular cartilage is to maintain the homeostasis between extracellular matrix (ECM) synthesis and catabolism, and reduce joint friction and impact (4). Chondrocytes synthesize and secrete components of the ECM infrastructure, in addition to various matrix-degrading enzymes, proteoglycan-degrading enzymes and other hydrolytic enzymes; this facilitates the degradation and digestion of denatured and dysfunctional ECM proteins, which alters the structure of the ECM (5). Therefore, apoptosis or abnormal physiological functioning of chondrocytes markedly changes the dynamic balance of ECM metabolism, and the integrity of the articular cartilage structure and function; this is a common cause of OA (6).

Nuclear factor-erythroid 2-related factor 1 (Nrf1) acts as a transcription factor belonging to the cap'n'collar (CNC) basic-region leucine zipper (bZIP) family, which serves as a crucial integrator of nuclear and mitochondrial interactions, modulating essential processes ranging from protein production to mitochondrial biogenesis (7-9). It also serves a prominent role in apoptosis $(10,11)$, suggesting that NRF1 may be an important target for mediating chondrocytes apoptosis.

MicroRNAs (miRNAs) are non-coding RNAs of 19-25 nucleotides in length that downregulate the expression of specific target genes through interacting with motifs found primarily in their 3'-untranslatedregions (UTRs) $(12,13)$. miRNAs participate in the regulation of various physiological activities, including cellular proliferation, development, differentiation and apoptosis $(14,15)$. Previous studies have 
demonstrated that miRNAs regulate the occurrence and development of OA through various mechanisms. miRNA (miR)-26a was reported to suppress the activation of the $\mathrm{NF}-\kappa \mathrm{B}$ signaling pathway to alleviate synovial inflammation and cartilage injury in OA rats (16). miR-10a-5p promoted chondrocyte apoptosis in OA by targeting homeobox A1 (17). miR-93 targets Toll-like receptor $4 / \mathrm{NF}-\kappa \mathrm{B}$ signaling to inhibit OA-associated inflammation and chondrocyte apoptosis (18). TargetScan database analysis predicted the existence of binding sites between NRF1 3'UTR and miR-363-3p (19); therefore, the present study investigated miR-363-3p. In addition, miR-363-3p is involved in apoptosis regulation (20). However, the biological role of miR-363-3p in chondrocyte apoptosis, and the relationship between miR-363-3p and NRF1 have not been described. The present study aimed to investigate whether miR-363-3p could target NRF1-regulated chondrocyte apoptosis in OA model rats in vivo, and in lipopolysaccharide (LPS)-treated chondrocytes in vitro.

\section{Materials and methods}

Animal studies. The Animal Care and Use Committee of Shandong University (Shandong, China) approved all animal studies, TargetScan database (release 7.2) (19) identified a putative miR-363-3p target site within the NRF1-3'UTR which were conducted in a manner consistent with the National Institutes of Health Guide for the Care and Use of Laboratory Animals. A total of 36 male Wistar rats (age, 6 weeks; weight, 180-200 g) were obtained from the Shandong Center for Disease Control and housed at $24 \pm 2{ }^{\circ} \mathrm{C}$ in $50 \pm 5 \%$ humidity, under a 12 -h light/dark cycle, with free access to food and water. The rats were randomized into six groups: i) Untreated control group $(n=6)$; ii) untreated OA group $(n=6)$; iii) $\mathrm{OA}+$ agomir/antagomir miR-363-3p negative control (NC) group $(n=6 / 6)$; iv) $\mathrm{OA}+$ agomir/antagomir miR-363-3p group $(n=6 / 6)$. The rats were anaesthetized with $2 \%$ sodium phenobarbital $(50 \mathrm{mg} / \mathrm{kg}$; i.p.) and the OA model was established by medial meniscectomy tear (MMT) surgery; rats in the untreated control group were subjected to control surgery. Then, 1 week after MMT surgery, rats in the OA + agomir/antagomir miR-363-3p and the $\mathrm{OA}+$ agomir/antagomir miR-363-3p NC groups were treated with intra-articular injections of $5 \mathrm{nmol}$ agomir/antagomir miR-363-3p or agomir/antagomir-NC (Suzhou GenePharma Co., Ltd.), delivered using a medial parapatellar approach. The rats which were subjected to MMT and treated with agomir-NC as the negative control. Rats in control group and OA group were normally bred, without any intervention. Following 2 weeks, all rats were sacrificed for the articular cartilages of the medial tibial plateau and the synovial fluid by intraperitoneal injection with pentobarbital sodium overdose $(150 \mathrm{mg} / \mathrm{kg})$, which were collected and stored at $-80^{\circ} \mathrm{C}$ for subsequent analysis. The tissues were subsequently dehydrated in graded concentrations of ethanol $(50,75,85,95$ and $100 \%)$, cleared in xylene, embedded in paraffin and sectioned into $4 \mu \mathrm{m}$ tissue sections.

Primary rat articular chondrocytes isolation and culture. Intact tibial plateau cartilage tissue was cut into 2-3 mm slices with a scalpel blade, and then 20 slices were digested in $0.25 \%$ trypsin (R\&D Systems, Inc.) at $37^{\circ} \mathrm{C}$ for $30 \mathrm{~min}$. Segments were washed twice using $1 \mathrm{X}$ PBS, followed by further digestion using $3 \mathrm{mg} / \mathrm{ml}$ collagenase D (Thermo Fisher Scientific, Inc.) in DMEM (Invitrogen; Thermo Fisher Scientific, Inc.) for $12 \mathrm{~h}$ at $37^{\circ} \mathrm{C}$ and cartilage tissue were completely digested and chondrocytes were clearly visible. The digested tissue was filtered through a sterile $150 \mu \mathrm{M}$ nylon mesh. At this point, collagen was isolated and underwent centrifugation at $600 \mathrm{xg}$ for $5 \mathrm{~min}$ at $4^{\circ} \mathrm{C}$. Subsequently, the pellet was suspended in DMEM and the undigested cartilage was removed by $70 \mathrm{~mm}$ nylon mesh. Then, the chondrocytes were cultured in a humidified atmosphere of $37^{\circ} \mathrm{C}$ and $5 \% \mathrm{CO}_{2}$ in $5 \mathrm{ml} \mathrm{DMEM}$ (Invitrogen; Thermo Fisher Scientific, Inc.) supplemented with 10\% FBS (Gibco; Thermo Fisher Scientific, Inc.) and 1\% penicillin-streptomycin. LPS-stimulation of chondrocytes is an established cellular model for the study of OA $(21,22)$. In the present study, when the cells reached $2 \times 10^{5} /$ well, chondrocyte apoptosis was induced by treatment with LPS (Sigma-Aldrich; Merck $\mathrm{KGaA}$ ) at a final concentration $5 \mu \mathrm{g} / \mathrm{ml}$ for $6 \mathrm{~h}$ (18).

Reverse transcription-quantitative PCR (RT-qPCR). TRIzol ${ }^{\circledR}$ reagent (Invitrogen; Thermo Fisher Scientific, Inc.) was used to isolate total RNA from chondrocytes $\left(5 \times 10^{6}\right)$ and articular cartilage RNA (50 mg), according to the manufacturer's protocol. Total RNA was quantified and subsequently reverse transcribed into cDNA using the Revert Aid First Strand cDNA Synthesis kit (cat. no. K1622; Thermo Fisher Scientific, Inc.), according to the manufacturer's protocol. The thermocycling conditions of qPCR were $25^{\circ} \mathrm{C}$ for $5 \mathrm{~min}, 42^{\circ} \mathrm{C}$ for $60 \mathrm{~min}$ and $70^{\circ} \mathrm{C}$ for $10 \mathrm{~min}$. qPCR was subsequently performed using the Applied Biosystems SYBR ${ }^{\circledR}$ Green Master mix (Thermo Fisher Scientific, Inc.) and an ABI StepOnePlus RT PCR platform (Thermo Fisher Scientific, Inc.) was used following the manufacturer's protocol. The following primers were used for the qPCR: miR-363-3p, forward 5'-GGATGCGGATGG GCGAGAGC-3', reverse 5'-TTAGCGGATGCGGAAAAT C-3'; NRF1, forward 5'-TTACTCTGCTGTGGCTGATGG-3', reverse 5'-CCTCTGATGCTTGCGTGGTCT-3'; Bcl-2, forward 5'-GGGACGCGAAGTGCTATTGGT-3', reverse 5'-CTCAGGCTGGAAGGAGAAGAT-3'; Bax, forward 5'-GGTTGCCCTCTTGTACTTTGC-3', reverse 5'-TCTTCC AGATGGTGAGCGAG-3'; p53, forward 5'-TTGCCGTCC CAAGCAATGGATGA-3', reverse 5'-TCTGGGAAGGGA CAGAAGATGAC-3'; cleaved caspase-3, forward 5'-ATG GAGAACAACAAAACCTCAGT-3', reverse 5'-TTGCTC CCATGTATGGTCTTTAC-3'; $\beta$-actin, forward 5'-CCCGCC GCCAGCTCACCATGG-3', reverse 5'-AAGGTCTCAAAC ATGATCTGGGTC-3' (Invitrogen; Thermo Fisher Scientific, Inc.). Relative miR-363-3p expression was normalized to U6, forward 5'-CGCTTCGGCAGCACATATACTA-3' and reverse 5'-CGCTTCACGAATTTGCGTGTCA-3'. mRNA expressions were normalized to the internal reference gene, $\beta$-actin; the expression levels were quantified using the $2^{-\Delta \Delta \mathrm{Cq}}$ method (23).

Cell transfection. NRF1 siRNAs and control siRNA were purchased from Invitrogen (Thermo Fisher Scientific, Inc.), and miR-363-3p agomir, agomir NC, miR-363 antagomir and antagomir NC were purchased from Suzhou GenePharma Co., Ltd. Once the chondrocytes reached $50 \%$ confluency, agomir and antagomir (100 $\mathrm{nM}$ in vitro, $40 \mathrm{nmol} / 200 \mu \mathrm{l}$ in vivo), and siRNA $(100 \mathrm{nM})$ were transfected with these constructs using Lipofectamine ${ }^{\circledR} 2000$ (Invitrogen; Thermo Fisher Scientific, 
Inc.), according to the manufacturer's protocol. Following $48 \mathrm{~h}$ of transfection at $37^{\circ} \mathrm{C}$, cells were harvested for further experimentation. siRNA oligonucleotide sequences were as follows: NRF1 siRNA (si-NRF1) forward, 5'-UUAAGCGCCAUA GUGACUG-3'; and control siRNA (si-NC): forward 5'-UAU UUGGAUGUACCUGUGGACUUGG-3'.

Cell viability assay. Chondrocyte viability was detected using the MTT assay. A total of $2 \times 10^{5}$ cells/well were seeded and transfected for $48 \mathrm{~h}$ of transfection. Following this incubation, $20 \mu \mathrm{l}$ MTT (Sigma-Aldrich, Merck KGaA) was added to each well for $6 \mathrm{~h}$. The purple formazan was dissolved in $200 \mu \mathrm{l}$ DMSO and the viability was analyzed using a Multiskan Spectrum microplate reader (Thermo Fisher Scientific, Inc.) at a wavelength of $450 \mathrm{~nm}$.

Flow cytometric analysis of apoptosis. Following $48 \mathrm{~h}$ of transfection, chondrocytes $\left(1 \times 10^{6}\right)$ were collected after centrifugation at $500 \mathrm{x} \mathrm{g}$ for $20 \mathrm{~min}$ at room temperature, to assess the apoptotic status. After washing the cells using $1 \mathrm{X}$ PBS, the chondrocytes were stained with Annexin $\mathrm{V}$ at $4^{\circ} \mathrm{C}$ for 15 mins, followed by an additional 5 min stain with propidium iodide from the Annexin V-FITC Apoptosis Detection kit (BD Biosciences), according to the manufacturer's protocol. Apoptotic cells were subsequently analyzed using flow cytometry, which was performed using the BD FACS Calibur Flow Cytometry Machine (BD Biosciences), and the results were analyzed using the FlowJo.7.6.1 software (BD Biosciences).

ELISA. Tumor necrosis factor (TNF)- $\alpha$, interleukin (IL)-1 $\beta$ and IL-6 expression levels in synovial fluid (100 $\mu \mathrm{l} /$ well), which was extracted by joint puncture, were analyzed using ELISA kits (TNF- $\alpha$, cat. no. RTA00; IL-1 $\beta$, cat. no. RLB00; IL-6, cat. no. R6000B; R\&D Systems, Inc.), according to the manufacturer's protocols.

Immunohistochemistry (IHC). NRF1, Bax, Bcl-2 and caspase-3 levels were measured by IHC. Briefly, tissues were fixed in $4 \%$ paraformaldehyde and subsequently paraffin embedded and cut into $4-\mu \mathrm{m}$-thick sections. After deparaffinized, the tissue sections were hydrated in graded ethanol, and then blocked with $3 \%$ hydrogen peroxide for $20 \mathrm{~min}$ at room temperature. Subsequently, sections were treated with $10 \%$ normal goat serum (cat. no. 16210072; Gibco; Thermo Fisher Scientific, Inc.) for $30 \mathrm{~min}$ at $37^{\circ} \mathrm{C}$. After that, the sections were incubated overnight at $4^{\circ} \mathrm{C}$ with the following primary antibodies (Abcam): Rabbit monoclonal anti-NRF1 (1:100; cat. no. ab175932), rabbit polyclonal anti-Bax (1:200; cat. no. ab53154), rabbit polyclonal anti-Bcl-2 (1:200; cat. no. ab196495), rabbit monoclonal anti-cleaved caspase 3 (1:200; cat.no. ab2302) and anti- $\beta$-actin (1:500; cat. no. ab8224). Following primary antibody incubation, sections were washed three times using $1 \mathrm{X}$ PBS, and incubated with horseradish peroxidase (HRP)-conjugated goat anti-rabbit IgG secondary antibodies (1:400; cat. no. G-21234; Thermo Fisher Scientific, Inc.) for $2 \mathrm{~h}$ at room temperature. The slides were subsequently stained with $1 \mathrm{mg} / \mathrm{ml} \mathrm{3,3'-diaminobenzidine} \mathrm{and} \mathrm{hematoxylin}$ counterstain for $10 \mathrm{~min}$ at room temperature. A light microscope (Olympus; model BX51) was used to capture the images (magnification, $\mathrm{x} 400$ ).
Immunofluorescence staining. Chondrocytes were fixed with $4 \%$ paraformaldehyde for $30 \mathrm{~min}$ at $4^{\circ} \mathrm{C}$ and subsequently permeabilized with $0.1 \%$ Triton $\mathrm{X}-100$ for $10 \mathrm{~min}$ at $4^{\circ} \mathrm{C}$. Following blocking in $10 \%$ donkey serum (Sigma-Aldrich; Merck KGaA) for 20 min at room temperature, the cells were incubated with primary antibodies (Abcam) targeting NRF1 (1:200; cat. no. ab175932), p53 (1:200; cat. no. ab131442), Bcl-2 (1:200; cat. no. ab196495) and cleaved caspase-3 (1:150; cat. no. ab2302) overnight at $4^{\circ} \mathrm{C}$. The samples were subsequently incubated with goat anti-rabbit $\operatorname{IgG}(\mathrm{H}+\mathrm{L})$ highly cross-adsorbed secondary antibodies (1:400; cat. no. A32731; Invitrogen; Thermo Fisher Scientific, Inc.) at room temperature for $30 \mathrm{~min}$. Nuclear staining was achieved using DAPI [Roche Diagnostics (Shanghai) Co., Ltd.]. A fluorescent microscope (Leica) was used to capture the images (magnification, x200 or $\mathrm{x} 400)$.

TUNEL assay. Articular cartilage tissue sections were fixed in $4 \%$ paraformaldehyde for $24 \mathrm{~h}$ at room temperature and embedded in paraffin. Then, $4 \mu \mathrm{m}$-thick paraffin sections were deparaffinized using 100\% xylene, rehydrated for 5 min twice at room temperature and washed with $\mathrm{H}_{2} \mathrm{O}$. This was followed by rehydrated with ethanol at graded concentrations $(100 \%$ $5 \mathrm{~min}, 100 \% 3 \mathrm{~min}, 95 \% 3 \mathrm{~min}, 85 \% 3 \mathrm{~min}, 70 \% 3 \mathrm{~min}, 50 \%$ $3 \mathrm{~min}$ ) at room temperature. Then, the tissues sections were treated with $100 \mu \mathrm{l}$ proteinase $\mathrm{K}$ [20 $\mu \mathrm{g} / \mathrm{ml}$; Roche Diagnostics (Shanghai) Co., Ltd.)] for $20 \mathrm{~min}$ at room temperature, and washed 1X PBS. Subsequently, chondrocyte apoptosis in the articular cartilage was measured using a In situ Cell Death Detection kit (cat. no. 11684817910; Roche Diagnostics Co., Ltd.), according to the manufacturer's protocols. Cells with brown nuclei were deemed TUNEL-positive and were counted by a microscope using three fields of view/section. A light microscope (Olympus Corporation; model BX51) was used to capture the images (magnification, $\mathrm{x} 200$ ).

Safranin $O$ staining. Sections of knee cartilage tissue $(4 \mu \mathrm{m})$ were fixed with $4 \%$ formaldehyde at $20^{\circ} \mathrm{C}$ for 30 mins, paraffin embedded and serially separated into $4 \mu \mathrm{m}$ sections. Sections were then stained with $0.1 \%$ Weigert's iron hematoxylin at room temperature for $5 \mathrm{~min}$ and washed by water for $1 \mathrm{~min}$ at room temperature. The sections were differentiated for $30 \mathrm{sec}$ in $1 \%$ ethylic acid solution, incubated in $0.2 \%$ fast green solution (Thermo Fisher Scientific, Inc.) for $1 \mathrm{~min}$ at room temperature and then rinsed with distilled water for $1 \mathrm{~min}$. The sections were incubated in $0.1 \%$ safranin $\mathrm{O}$ solution (Thermo Fisher Scientific, Inc.) for $2 \mathrm{~min}$ at room temperature. The Safranin O solution (0.1-0.5 mg/ml; prepared in $\mathrm{H}_{2} \mathrm{O}$ ) was used as a counterstain and staining occurred at room temperature for $5 \mathrm{~min}$. A light microscope (Olympus Corporation; model BX51) was used to capture the images (magnification x100). After the Safranin O staining, the degree of articular cartilage lesions was scored by three independent observers according to the modified Mankin scoring principle (24). The score range was $0-14$, and the higher the score, the more severe the joint injury.

Dual-luciferase reporter assay. TargetScan database (release 7.2) (19) identified a putative miR-363-3p target site within the NRF1-3' untranslated region (UTR). Wild-type (WT) and mutant (MT) versions of the NRF1 candidate miR-363-3p 
A
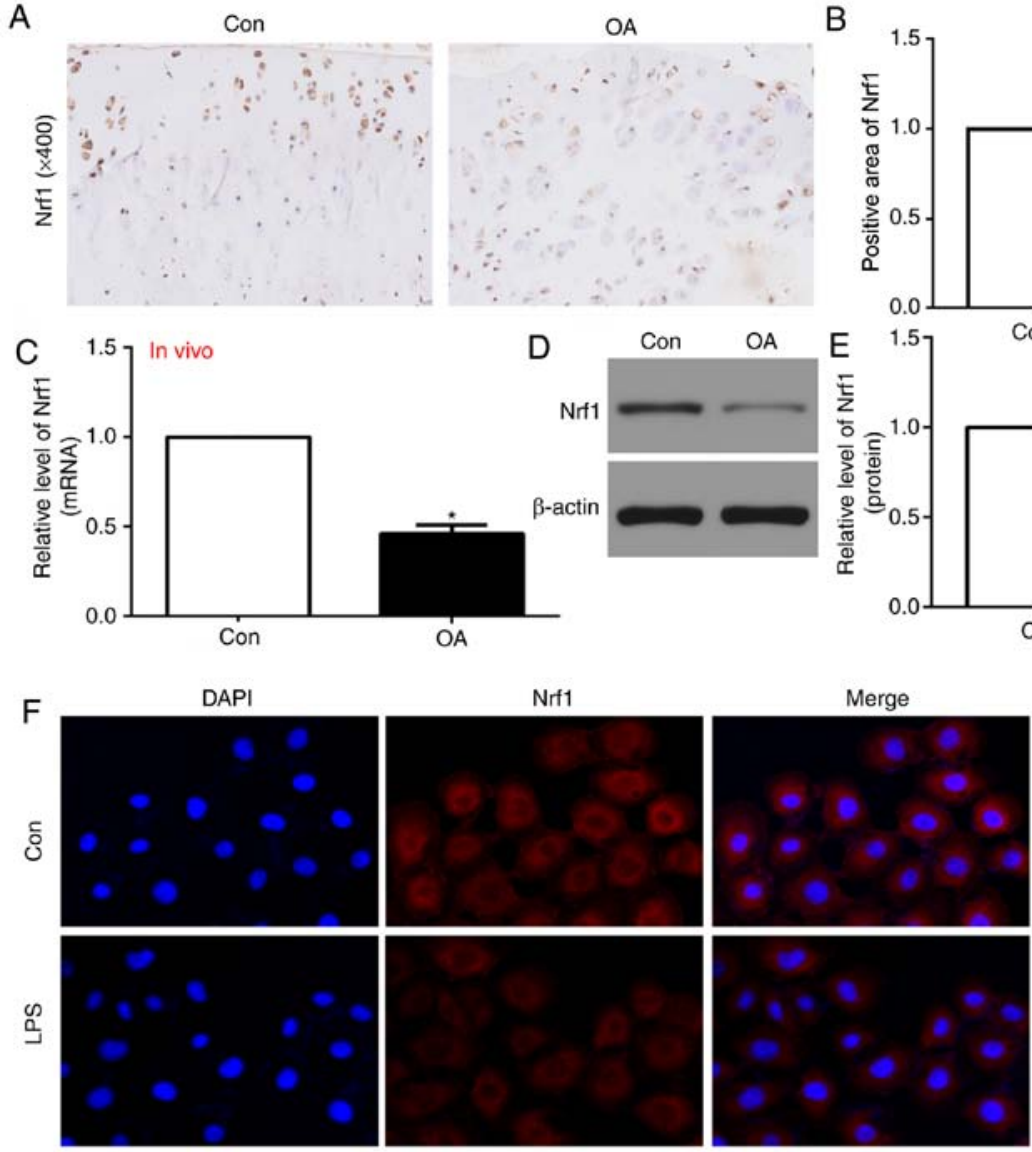

$\mathrm{H}$

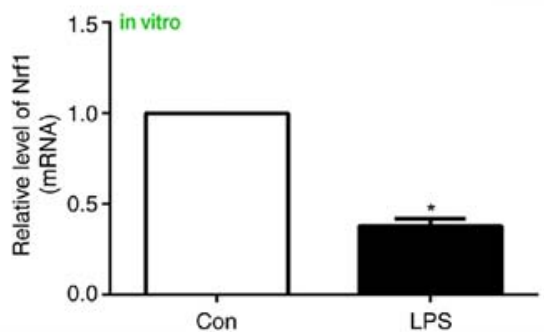

I

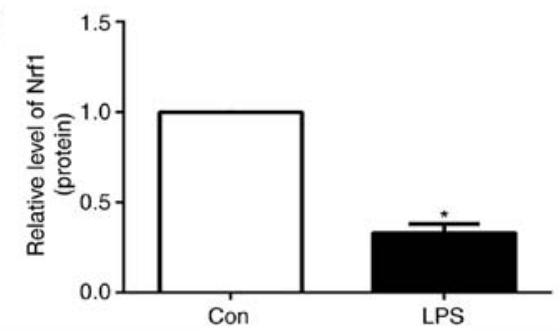

B
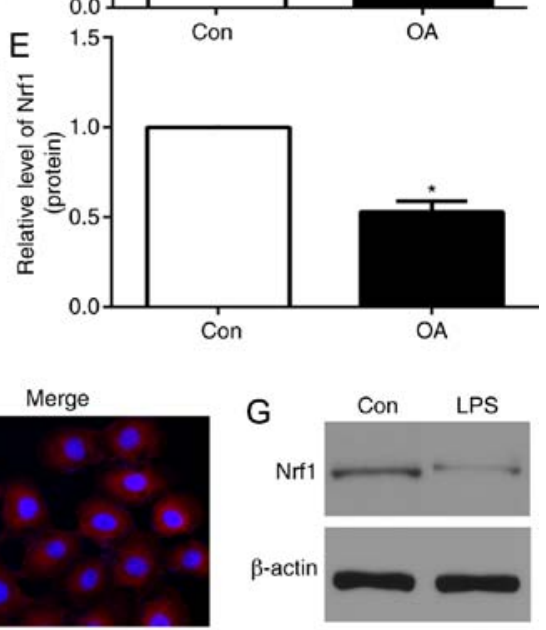

Figure 1. NRF1 is downregulated in OA model rats and LPS-treated chondrocytes. (A) In vivo NRF1 expression of chondrocytes was assessed by immunohistochemistry staining (magnification, $\mathrm{x} 400$ ) and (B) quantification (brown area \%) in the OA and control group (n=6 rats/group). In vivo mRNA and gene expression levels of NRF1 were assessed by (C) RT-qPCR and (D) western blot analysis in the OA and untreated control group (n=6 rats/group). (E) In vitro immunofluorescence of NRF1 expression in LPS-treated chondrocytes compared with the untreated control group (magnification, $\mathrm{x} 400$ ). (F) Western blotting results of NRF1 expression level in LPS-treated chondrocytes. (H) mRNA and (G and I) protein expression levels of NRF1 were assessed by RT-qPCR and western blot analysis, respectively, in LPS-treated chondrocytes. "P<0.05 vs. control group. RT-qPCR, reverse transcription-quantitative PCR; LPS, lipopolysaccharide; OA, osteoarthritis; NRF1, nuclear respiratory factor 1 ; Con, untreated control.

target sequences were generated and cloned into the pGL3 vector bearing a firefly luciferase reporter element (Promega Corporation), yielding wild-type (wt-pGL3-NRF1-3'UTR) or mutant (mut-pGL3-NRF1-3'UTR) constructs. 293T cells (American Type Culture Collection) were plated into 24-well plates $\left(2 \times 10^{5} /\right.$ well $)$ and subsequently transfected with the wt-pGL3-NRF1-3'UTR or mut-pGL3-NRF1-3'UTR constructs using Lipofectamine ${ }^{\circledR} 2000$ (Invitrogen; Thermo Fisher Scientific, Inc.), according to the manufacturer's protocol. Following incubation for $48 \mathrm{~h}$, cells were collected and the luciferase activity was detected using a Dual-Luciferase Reporter assay system (Promega Corporation). Scramble miRNA was used as a negative control, and the Renilla luciferase intensity as normal control, according to the manufacturer's protocol.
Western blotting. Intact tibial plateau cartilage tissue or chondrocytes $\left(5 \times 10^{6}\right)$ protein were extracted on ice using a lysis buffer supplemented with protease inhibitors [Roche Diagnostics (Shanghai) Co., Ltd.] for $50 \mathrm{~min}$ at $4^{\circ} \mathrm{C}$ and centrifuged for $15 \mathrm{~min}$ at $12,000 \mathrm{x} \mathrm{g}$. Total protein levels were quantified using a bicinchoninic acid assay kit (Thermo Fisher Scientific, Inc.) The protein samples were diluted in 5X sample buffer (ratio, 4:1; cat. No. MB01015; GenScript) and incubated for $5 \mathrm{~min}$. A total of $40 \mu \mathrm{g}$ of proteins was separated by $10 \%$ SDS-PAGE gel. The PVDF membrane was blocked with TBS containing 5\% non-fat milk and $0.1 \%$ Tween for $1 \mathrm{~h}$ at room temperature. Following blocking, membranes were incubated overnight at $4{ }^{\circ} \mathrm{C}$ with primary antibodies (Abcam) against NRF1 (1:100; cat. no. ab175932), p53 (1:200; cat. no. ab131442), 


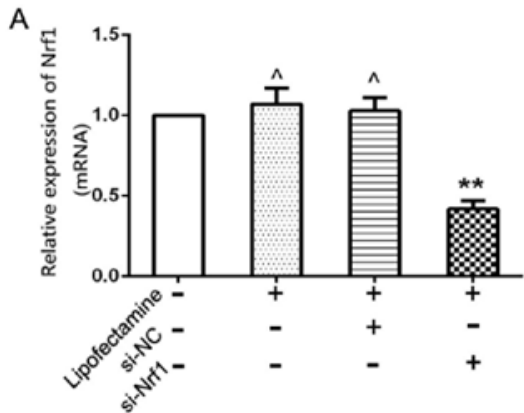

C

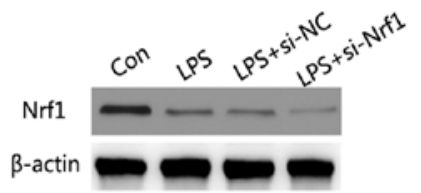

B
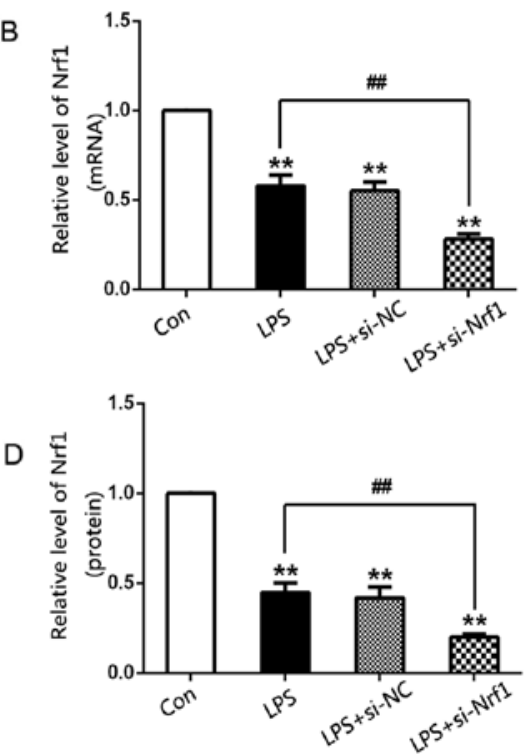

$\mathrm{E}$
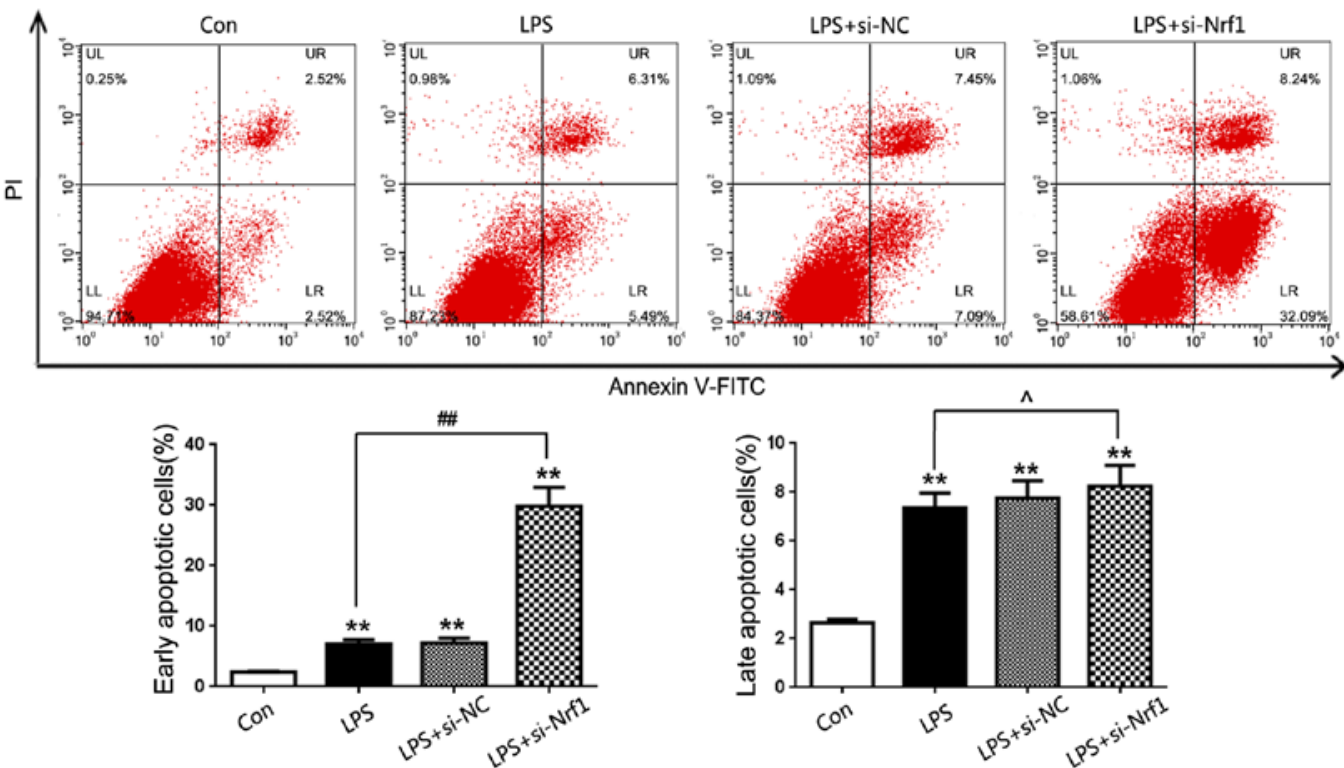

Figure 2. Influence of NRF1 on LPS-induced chondrocyte apoptosis in vitro. (A) Relative mRNA expression levels of NRF1 were determined by RT-qPCR analysis in cultured chondrocytes following the genetic knockdown of NRF1 with siRNA ( $n=6 /$ group). (B) mRNA expression levels of NRF1 were assessed in LPS-treated chondrocytes by RT-qPCR. (C) Western blot analysis and (D) quantification following the genetic knockdown of NRF1 with siRNA (E) Flow cytometry analysis was performed to determine cellular apoptosis following the genetic knockdown of NRF1 with siRNA in LPS-treated chondrocytes. ${ }^{* *} \mathrm{P}<0.01$ vs. con, ${ }^{\# \#} \mathrm{P}<0.01,,{ }^{\wedge}>0.05$ vs. LPS group. RT-qPCR, reverse transcription-quantitative PCR; LPS, lipopolysaccharide; OA, osteoarthritis; NRF1, nuclear respiratory factor 1; siRNA, small interfering RNA; NC, negative control; si/siRNA, small interfering RNA; Con, untreated control; PI, propidium iodide.

cleaved caspase 3 (1:200; cat. no. ab2302) and $\beta$-actin (1:500; cat. no. ab8224). Following the primary incubation, membranes were incubated at room temperature for $1 \mathrm{~h}$ with HRP-conjugated secondary antibodies (1:500; cat. no. A20207; Invitrogen; Thermo Fisher Scientific, Inc.). Protein bands were visualized and detected using Odyssey Infrared Imaging system Model 9120 (LI-COR Biotechnology) with Quantity One software (version 2.4; Bio-Rad Laboratories, Inc.). $\beta$-actin was used for normalization.

Statistical analysis. Statistical analysis was performed using the SPSS version 22.0 (IBM Corp.) software, and data are presented as the mean \pm SD. All experiments were performed $\geq 3$ times. Statistical significance between groups was determined using Student's t-test or one-way ANOVA with Tukey's post hoc test. Simple correlations were assessed by using the Pearson correlation. The target genes of NRF1 were predicted with TargetScan. $\mathrm{P}<0.05$ was considered to indicate a statistically significant difference.

\section{Results}

NRF1 expression is reduced in the articular cartilage of $O A$ rats in vivo and LPS-treated chondrocytes in vitro. To investigate the potential involvement of NRF1 in OA, the gene and protein expression levels were investigated in vivo by RT-qPCR and western blotting, respectively, in addition to IHC in vitro. The mRNA and protein expression levels of 

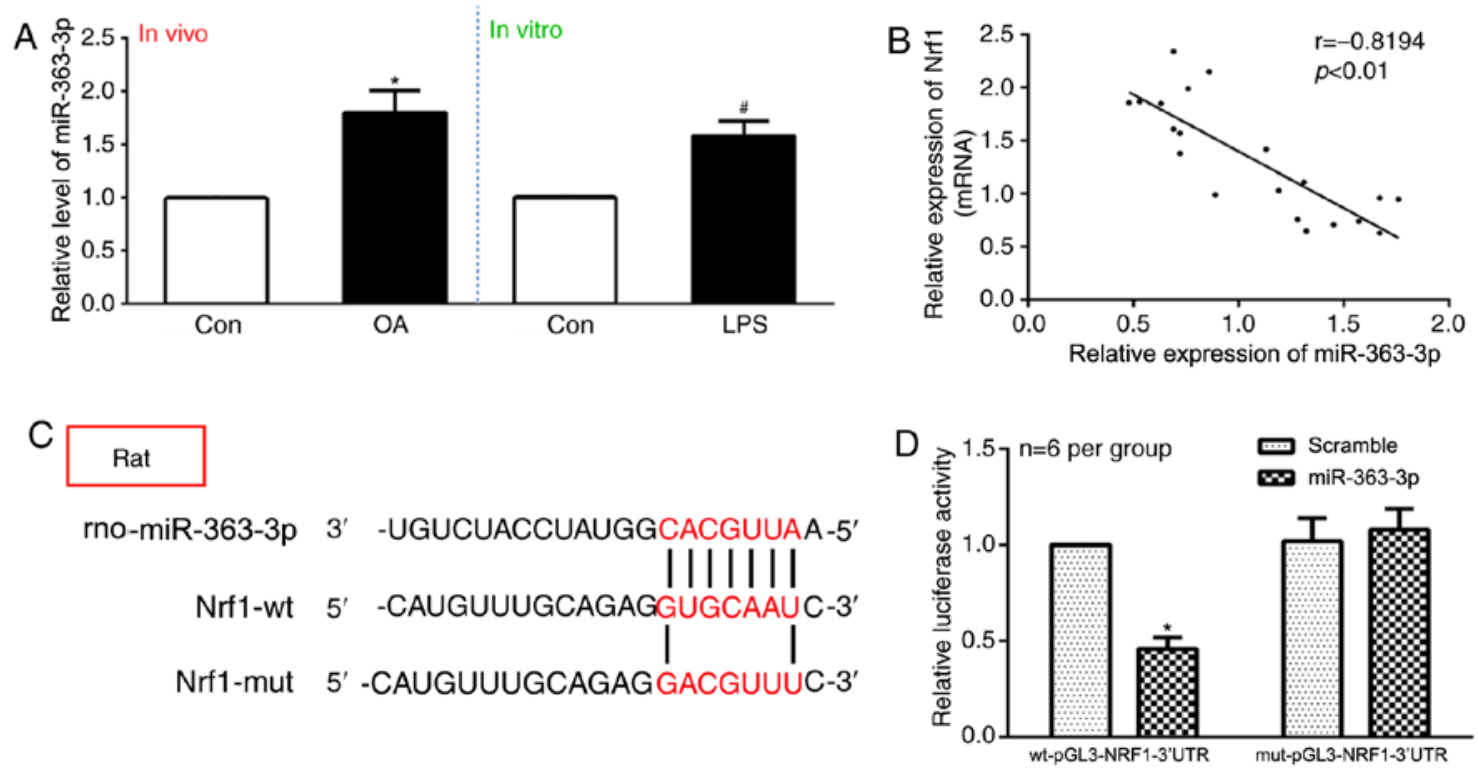

Figure 3. NRF1 is a direct target of miR-363-3p. (A) Expression levels of miR-363-3p were evaluated by RT-qPCR in OA rats compared with the control group (in vivo), and LPS-treated chondrocytes compared with control chondrocytes (in vitro) ( $\mathrm{n}=\mathrm{rats} 6$ 6roup). (B) Pearson's correlation analysis revealed that the mRNA expression of miR-363-3p was inversely correlated with the expression of NRF1 (P<0.01). (C) TargetScan database predicted the existence of binding sites between NRF13'UTR and miR-363-3p. The mutation was generated on the NRF1 3'UTR sequence in the complementary site for the binding region of miR-363-3p. (D) Dual-reporter luciferase assay of miR-363-3p expression in wt-pGL3-NRF1-3'UTR or mut-pGL3-NRF1-3'UTR constructs. "P<0.05 vs. control group, Wt, wild-type; mut, mutant; miR, microRNA; OA, osteoarthritis; NRF1, nuclear respiratory factor 1; UTR, untranslated region; RT-qPCR, reverse transcription-quantitative PCR; NC, negative control; LPS, lipopolysaccharide; Scramble, scramble-miRNA.

NRF1 were revealed to be downregulated within the articular cartilage of OA model rats compared with the control group (Fig. 1A-E). Consistent with this, LPS-treated chondrocytes exhibited reduced NRF1 mRNA and protein expression compared with untreated (control) chondrocytes (Fig. 1F-I). These data demonstrated that NRF1 may be important in OA.

Influence of NRF1 on chondrocyte apoptosis in vitro. To investigate how NRF1 affected LPS-induced chondrocyte injury, siRNA-NRF1 was used to knockdown NRF1 gene expression levels in the chondrocytes. siRNA-NRF1 significantly decreased NRF1 gene expression levels in chondrocytes relative to the si-NC group, demonstrating an effective transfection efficiency (Fig. 2A). In addition, NRF1 significantly decreased the mRNA and protein expression levels in LPS-treated chondrocytes compared with the NCs (Fig. 2B-D). Flow cytometric analysis confirmed that LPS induced apoptosis in chondrocytes and that chondrocytes transfected with si-NRF1 further promoted LPS-induced early apoptosis rather than late apoptosis (Fig. 2E). This indicated that NRF1 may prevent apoptosis in vitro.

miR-363-3p and NRF1 expression are negatively correlated in vivo, and miR-363-3p targets NRF1 in vitro. miR-363-3p expression levels in vivo were significantly increased in the OA group compared with the control group, and in the LPS-treated chondrocytes compared with the control chondrocytes in vitro (Fig. 3A). The correlation between miR-363-3p and NRF1 expression was assessed using Pearson's correlation analysis, which revealed that NRF1 mRNA expression was negatively correlated with miR-363-3p expression in vivo (Fig. 3B). TargetScan database analysis identified a putative miR-363 target site within the NRF1-3'UTR (Fig. 3C). A dual-luciferase reporter approach was used to confirm this predicted binding site, using luciferase reporter constructs bearing wt-pGL3-NRF1-3'UTR or mut-pGL3-NRF1-3'UTR constructs. miR-363-3p overexpression led to a significant decrease in wt-pGL3-NRF1-3'UTR reporter activity, but not in mut-pGL3-NRF1-3'UTR activity, which demonstrated similar activity to the groups of scramble miRNA (Fig. 3D). The present results suggested that NRF1 may be a direct target of miR-363-3p.

miR-363-3p promotes apoptosis in OA model rats. To assess the functional relevance of miR-363-3p in OA development, OA model rats were intra-articularly administered agomir miR-363-3p. Expression of miR-363-3p in OA rats was significantly increased compared with the control groups. Significantly elevated miR-363-3p expression levels were demonstrated in the cartilage of OA + agomir miR-363-3p rats compared with the OA groups (Fig. 4A). Levels of pro-inflammatory cytokines in these rats were determined by ELISA, which revealed that TNF- $\alpha$, IL- $1 \beta$ and IL- 6 levels in OA rats was significantly increased compared to the control groups, and significantly increased in the agomir miR-363-3p OA group compared with $\mathrm{OA}$ and control groups (Fig. 4B-D). Furthermore, the effect of miR-363 on the articular cartilage of OA rats was analyzed by Safranin $O$ and TUNEL staining. The OA group treated with agomir miR-363-3p exhibited a significantly damaged surface cartilage layer, and the tide line was distorted and moved forward compared with the OA group, as revealed by Safranin $\mathrm{O}$ staining (Fig. 4E). In addition, compared with the OA group, agomir miR-363-3p increased the number of positive dark-brown stained cells, which were bigger compared with those in the OA group (Fig. 4E). Mankin's score of articular cartilage was previously evaluated by light microscopy, 

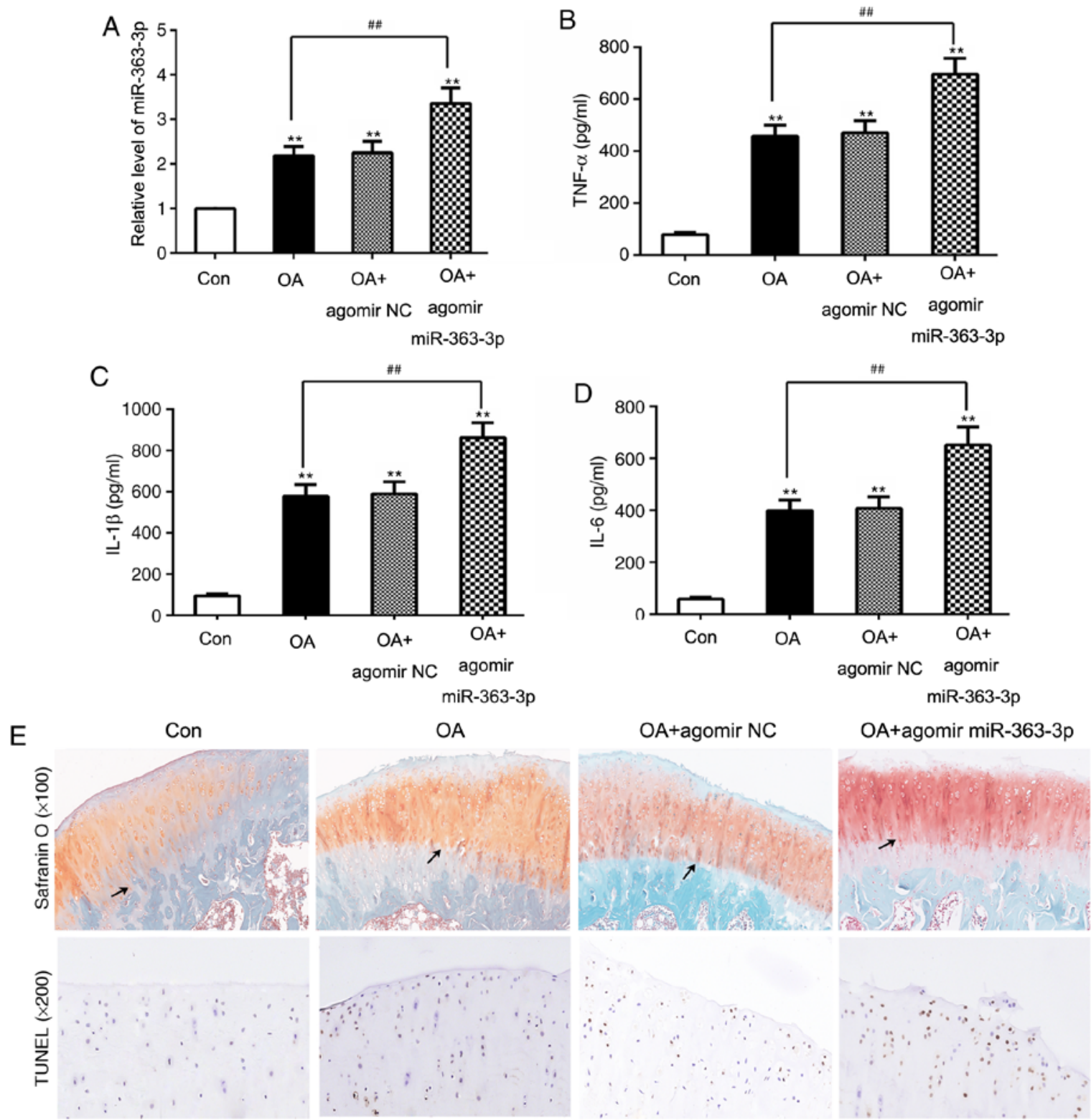

Figure 4. miR-363-3p promotes apoptosis in OA model rats. (A) Relative expression levels of miR-363-3p in OA rats injected with agomir miR-363-3p or agomir NC determined by reverse transcription-quantitative PCR analysis ( $\mathrm{n}=6$ rats/group). Release of pro-inflammatory cytokines (B) TNF- $\alpha$, (C) IL-1 $\beta$ and (D) IL-6 was measured by ELISA in OA rats injected with an agomir miR-363-3p compared to control groups. (E) Histological structure of articular cartilage in OA model rats injected with an agomir miR-363 compared with control groups were observed by Safranin O staining (magnification, x200). Apoptotic cells were examined by TUNEL assay (magnification, $\mathrm{x} 200$ ). ${ }^{* *} \mathrm{P}<0.01$ vs. control group, ${ }^{* \#} \mathrm{P}<0.01$ vs. OA group. OA, osteoarthritis; NC, negative control; TNF- $\alpha$, tumor necrosis factor- $\alpha$; IL, interleukin; miR, microRNA.

as previously described; compared with the control group $(0.33 \pm 0.52 ; n=6)$, significant pathological changes of articular cartilage in the OA group $(4.50 \pm 1.05 ; \mathrm{n}=6)$ and $\mathrm{OA}+$ agomir $\mathrm{NC}$ group $(5.00 \pm 0.89 ; \mathrm{n}=6)$ were observed. Agomir miR-363-3p $(8.50 \pm 1.05 ; \mathrm{n}=6)$ significantly increased the Mankin's score of OA articular cartilage. In addition, compared with the OA group, agomir miR-363-3p increased the number of positive dark-brown stained cells, which were bigger compared with those in the OA group (Fig. 4E). Taken together, these data suggested that miR-363-3p aggravated LPS-stimulated apoptosis and proinflammatory cytokine production.

miR-363-3p targets NRF1 and promotes LPS-induced chondrocyte apoptosis via the upregulation of p53 in vitro. First of all, the present study detected target gene expression by RT-qPCR, which showed the transfections of agomir and antagomir miR-363-3p were effective (Fig. 5A and B). To further investigate whether miR-363-3p regulates chondrocyte apoptosis, the chondrocytes transfected with agomirmiR-363-3p were stimulated with LPS, and the overexpression of miR-363-3p in the chondrocytes was evaluated using RT-qPCR analysis (Fig. 5A). The present results suggested that the expression level of miR-363-3p was significantly increased in the LPS group compared with the control group. In addition, agomir miR-363-3p further upregulated miR-363-3p expression in LPS-induced chondrocytes (Fig. 5C). Subsequently, the MTT assay to assess chondrocyte viability revealed that miR-363 overexpression significantly reduced chondrocyte viability compared with the control groups 

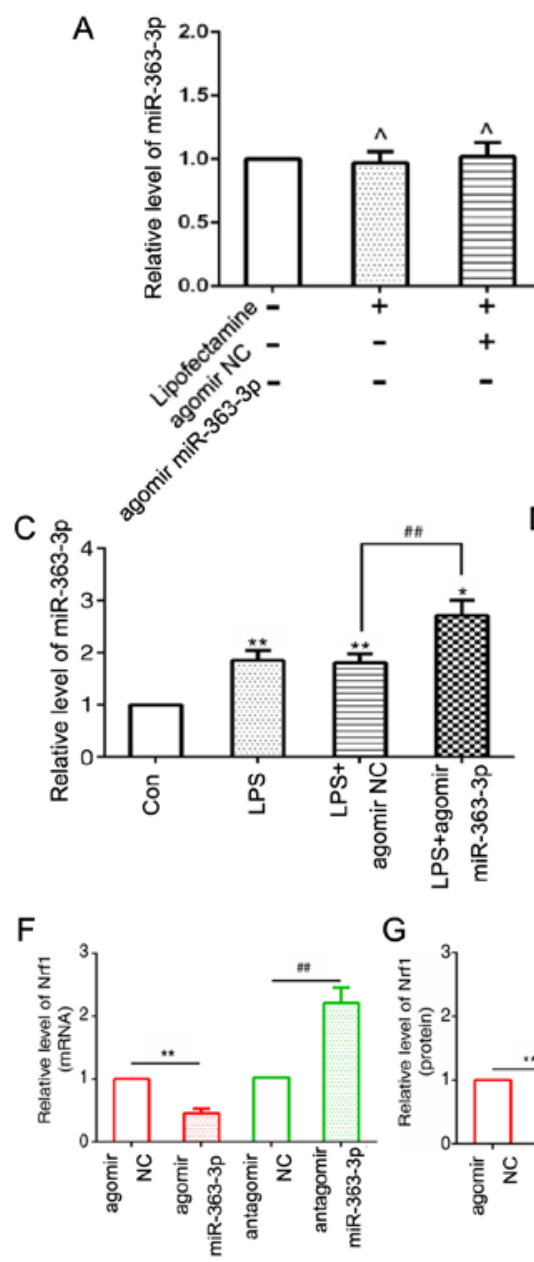

$\mathrm{J}$
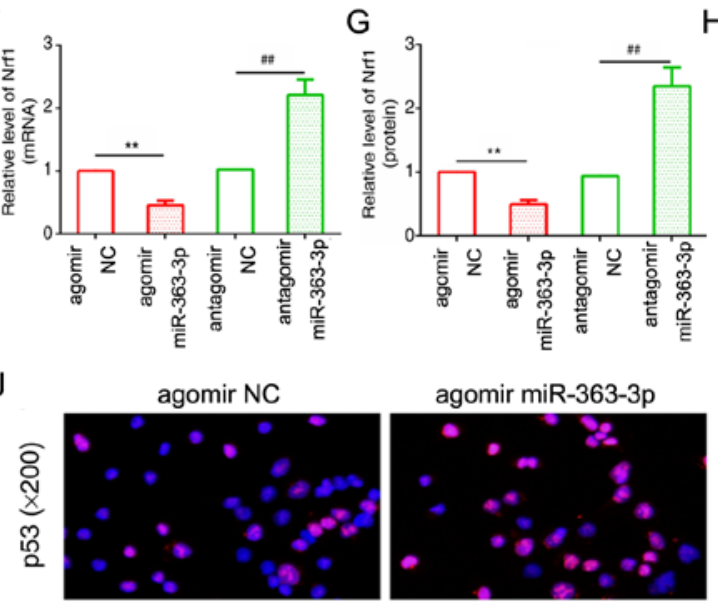

agomir miR-363-3p
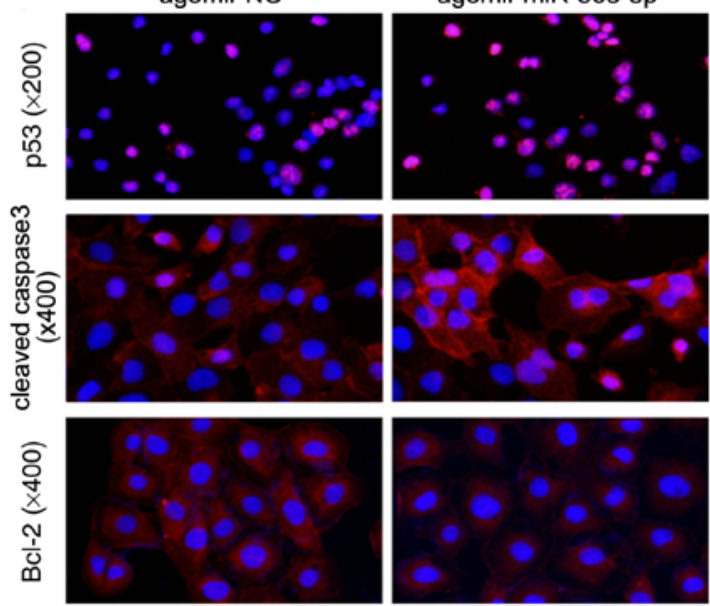

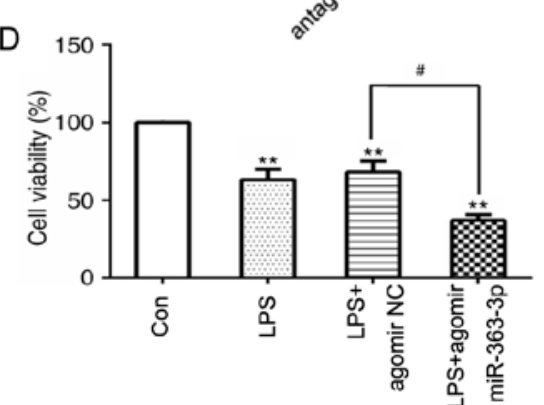

$\mathrm{H}$
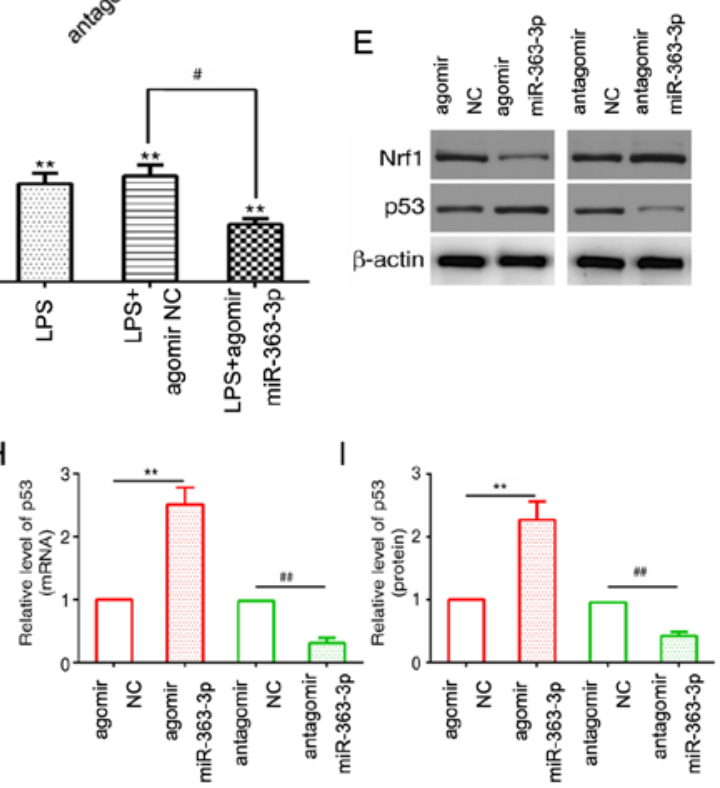

antagomir NC

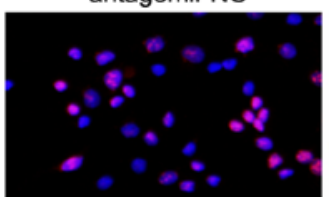

antagomir miR-363-3p
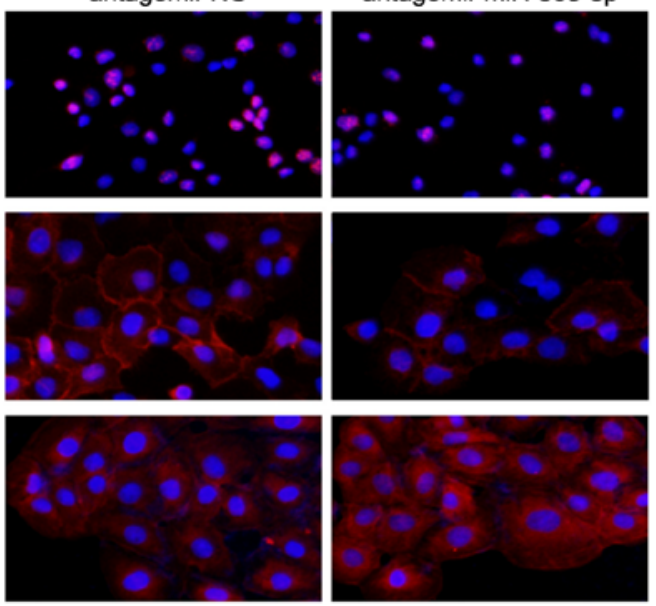

Figure 5. miR-363-3p enhances LPS-induced chondrocyte apoptosis and promotes the expression of p53 in vitro. (A and B) Transfection efficiency of agomir and antagomir miR-363-3p, was determined in cultured chondrocytes. (C) Relative expression levels of miR-363-3p were determined by RT-qPCR analysis in agomir miR-363-3p transfected chondrocytes stimulated with LPS compared with control groups. (D) Cell viability was measured in agomir miR-363-3p transfected chondrocytes stimulated with LPS compared with control groups using an MTT assay. (E) NRF1 and p53 protein expression was assessed by western blot analysis in the agomir or antagomir miR-363-3p transfected chondrocytes compared with their respective NCs. (F) mRNA and (G) protein expression level of NRF1, and (H) mRNA and (I) protein expression level of p53 following transfection of chondrocytes with agomir or antagomir miR-363-3p compared with their respective NCs. (J) Protein expression levels of p53 (magnification, x200), cleaved caspase-3 (magnification, x400) and Bcl-2 (magnification, $\mathrm{x} 400$ ), were assessed by immunofluorescence in chondrocytes transfected with agomir or antagomir miR-363-3p, compared with their respective NCs. ${ }^{* *} \mathrm{P}<0.01,,{ }^{,} \mathrm{P}<0.05,{ }^{\wedge} \mathrm{P}>0.05$ vs. control group, ${ }^{\# \prime} \mathrm{P}<0.01$ vs. LPS + miR-363-3p group, ${ }^{\prime} \mathrm{P}<0.05$ vs. LPS+miR-363 NC group. miR, microRNA; LPS, lipopolysaccharide; RT-qPCR, reverse transcription-quantitative PCR; NRF1, nuclear respiratory factor 1; NC, negative control; Con, control.

(Fig. 5D). Furthermore, miR-363-3p overexpression significantly downregulated NRF1 mRNA and protein expression levels compared with the agomir NC, whereas chondrocytes transfected with a miR-363-3p antagomir significantly increased NRF1 mRNA and protein expression compared with the antagomir NC (Fig. 5E-G). Expression levels of p53, cleaved caspase-3 

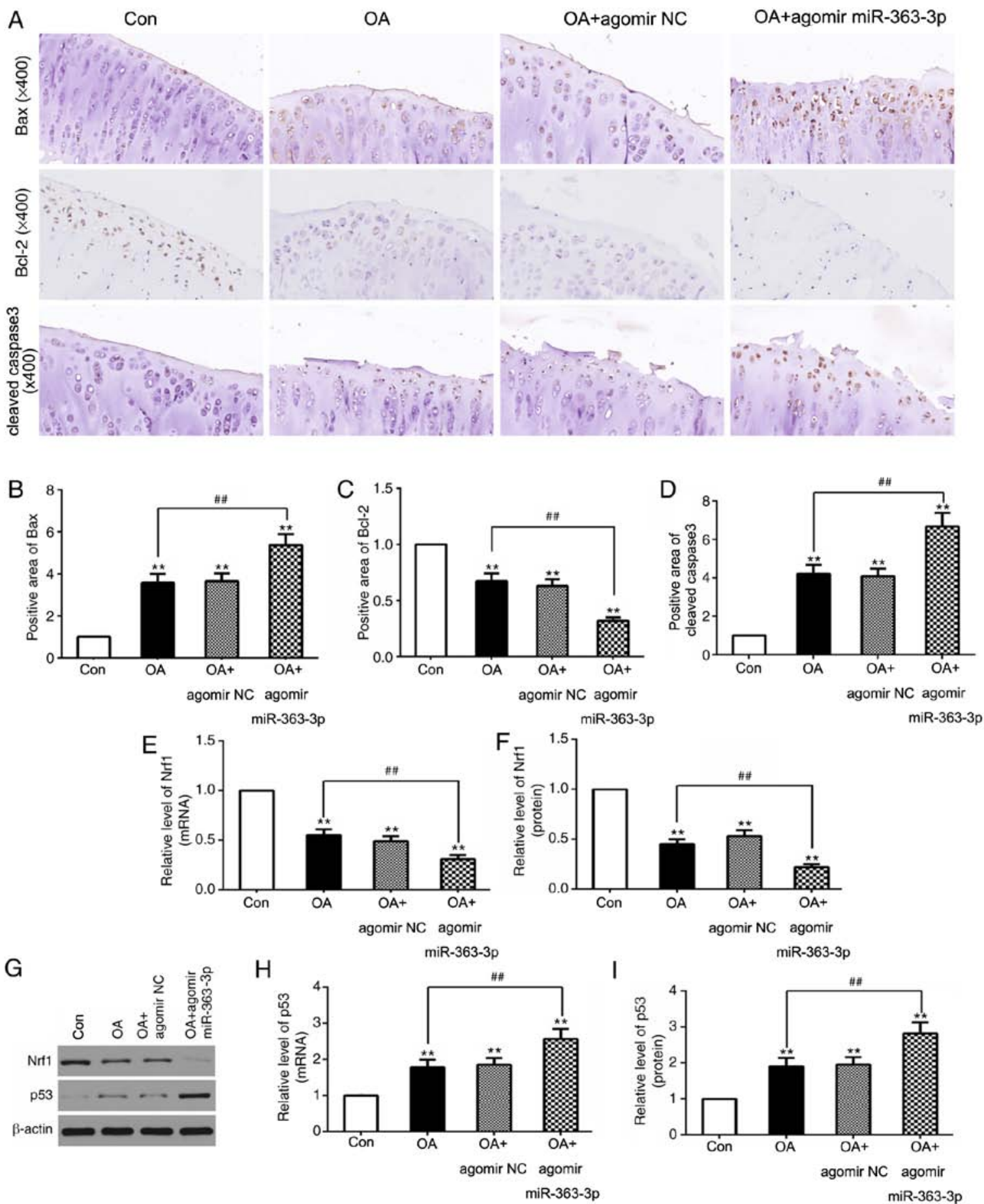

Figure 6. miR-363-3p targeting of NRF1regulates the expression of apoptotic index in chondrocytes of OA rats in vivo. (A) Immunohistochemistry staining in chondrocytes and quantification (brown area \%; magnification, $\mathrm{x} 400$ ) from the sections of (B) Bax, (C) Bcl-2 and (D) cleaved caspase-3, following injection of agomir miR-363-3p, or agomir NC (n=6 rats/group). Reverse transcription-quantitative PCR was used to analyze the mRNA levels of (E) NRF1 and (H) p53 following injection with agomir miR-363-3p or its NC. (G) Protein expression levels of (F) NRF1 and (I) p53 following injection with agomir miR-363-3p, or its $\mathrm{NC}$, as assessed by reverse transcription-quantitative $\mathrm{PCR}$ ( $\mathrm{n}=6$ rats/group). ${ }^{* *} \mathrm{P}<0.01$ vs. control, ${ }^{\# \#} \mathrm{P}<0.01$ vs. OA group. miR, microRNA; $\mathrm{NRF} 1$, nuclear respiratory factor 1; OA, osteoarthritis; $\mathrm{NC}$, negative control.

and Bcl-2, were assessed through immunofluorescence, and p53 expression was also assessed through western blotting and RT-qPCR (Fig. 5E, H and I). The overexpression of miR-363-3p in the chondrocytes significantly increased mRNA and protein expression levels of p53 and cleaved caspase-3, and reduced the expression of Bcl-2 compared with the respective NC chondrocytes (Fig. 5J). Antagomir miR-363 reduced expression levels of p53 and cleaved caspase-3, and increased Bcl-2 expression levels in chondrocytes (Fig. 5E, H-J). The present results suggested that targeting NRF1 promoted LPS-induced chondrocyte apoptosis in vitro.

miR-363-3p targeting of NRF1 promotes chondrocyte apoptosis via the upregulation of $p 53$ in OA model rats. To further 

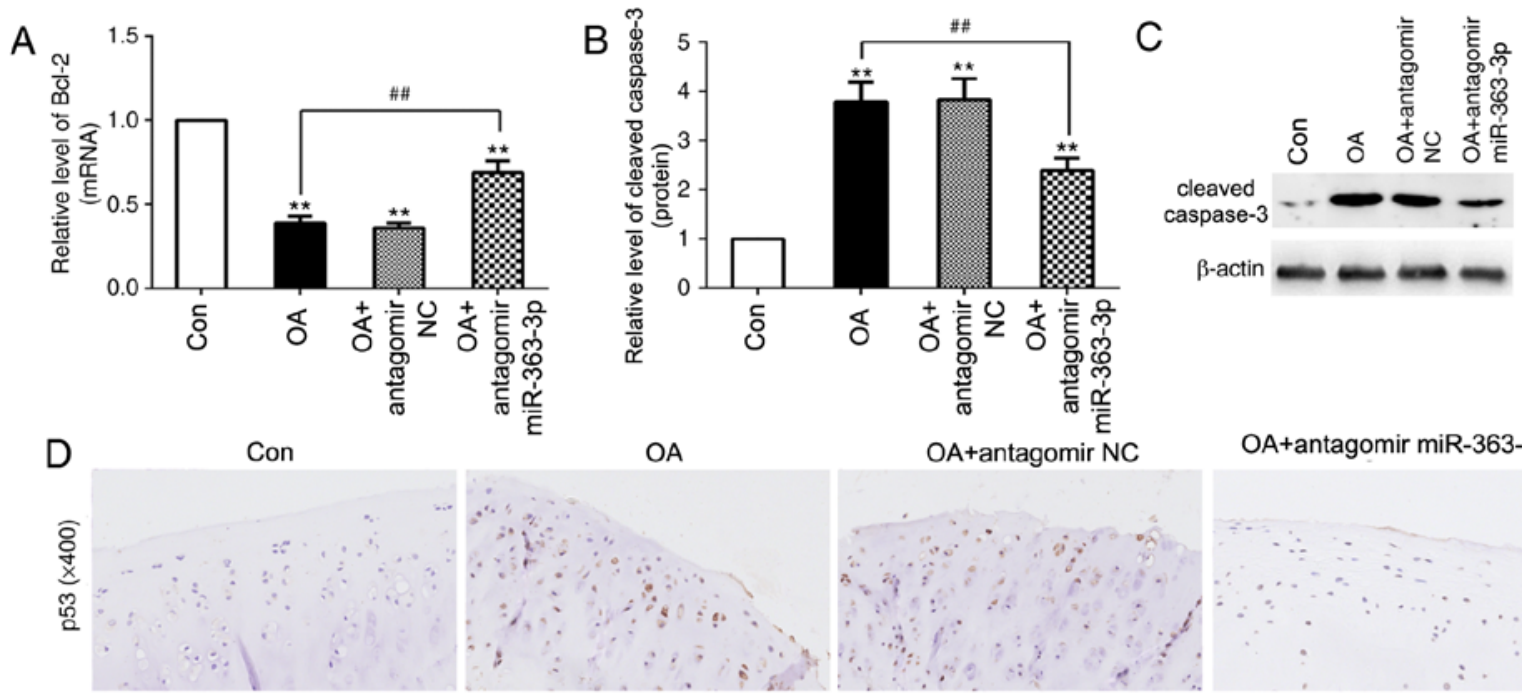

OA+antagomir miR-363-3p

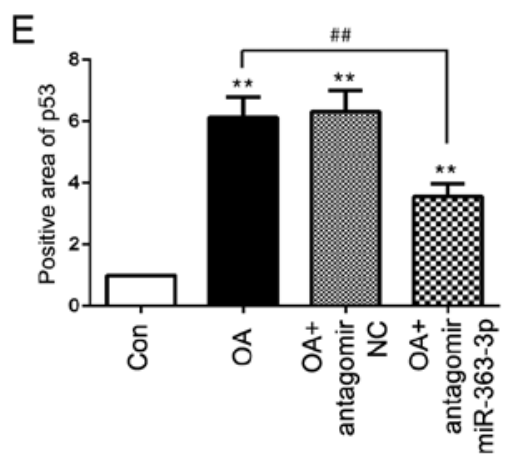

Figure 7. Inhibition of miR-363-3p upregulates Bcl-2 expression level, and downregulates cleaved caspase-3 and 53 expression levels in OA rats in vivo. (A) Reverse transcription-quantitative PCR assays were performed to analyze the mRNA expression levels of Bcl-2. (B and C) Western blot analysis of cleaved caspase-3 protein expression following injection with the agomir miR-363-3p ( $\mathrm{n}=6$ rats/group). (D) Immunohistochemistry staining (magnification, $\mathrm{x} 400$ ) in chondrocytes and (E) quantification (brown area \%) from the sections of p53 following injection with the agomir miR-363-3por agomir NC, in the OA rat model ( $\mathrm{n}=6$ rats/group). ${ }^{* * *} \mathrm{P}<0.01$ vs. control group, ${ }^{\# \#} \mathrm{P}<0.01$ vs. OA group. $\mathrm{NC}$, negative control; miR, microRNA; OA, osteoarthritis.

confirm that miR-363-3p regulates chondrocyte apoptosis in OA, in vivo experiments were conducted. miR-363-3p overexpression in OA model rats resulted in a significant increase of Bax and cleaved caspase- 3 expression, and a decrease of Bcl-2 expression (Fig. 6A-D), which suggested that miR-363-3p may promote chondrocyte apoptosis in OA. Furthermore, NRF1 mRNA and protein levels were decreased, and p53 mRNA and protein levels were increased following the overexpression of miR-363-3p with agomir miR-363 (Fig. 6E-I). These results provided further evidence that miR-363-3p targeting of NRF1 increased chondrocyte apoptosis through the upregulation of $\mathrm{p} 53$ in vivo.

Inhibition of $m i R-363-3 p$ reduces chondrocyte apoptosis in $O A$ rats. Based on the increased miR-363-3p expression levels demonstrated in vivo, whether the inhibition of miR-363-3p with antagomir miR-363 could reduce chondrocyte apoptosis was investigated. Compared with the $\mathrm{OA}+$ antagomir NC group, the OA + antagomir miR-363-3p demonstrated an increased mRNA expression of Bcl-2 and decreased protein expression of cleaved caspase-3 (Fig. 7A-C), which suggested that the inhibition of miR-36-3-3p may reduce chondrocyte apoptosis in OA. Furthermore, compared with the OA group, antagomirmiR-363-3p decreased the expression level of p53 in OA rats (Fig. 7D and E). These results suggested that inhibition of miR-363-3p may reduce chondrocyte apoptosis through the downregulation of $\mathrm{p} 53$ in vivo.

\section{Discussion}

OA is associated with articular cartilage degeneration and cartilage extracellular matrix destruction (25). The extrachondral matrix of the articular cartilage is mainly secreted by chondrocytes (26). In OA, a combination of multiple factors is directly or indirectly involved in cartilage matrix synthesis or metabolism, which ultimately results in OA-associated degradation of the cartilage (27). In osteoarthritic cartilage, the rate of chondrocyte loss is $20 \%$ (28). This indicates that chondrocytes are important for maintaining extrachondral matrix homeostasis and that the abnormal behavior of chondrocytes leads to the degeneration of the articular cartilage. An improved understanding of the molecular mechanisms governing the apoptotic death of chondrocytes in OA will therefore prove invaluable as a means of identifying novel therapeutic strategies to treat this disease.

In addition to regulating mitochondrial transcription and replication-associated gene expression, NRF1 is involved in the regulation of apoptosis. The knockdown of NRF1 led to increased apoptotic rates, which was likely associated with mitochondrial cytochrome $c$ release, and upregulated expression levels of Bax, caspase-3 and caspase-9, which are linked with apoptosis (29). The overexpression of NRF1 inhibited palmitate-induced human cardiac myocytes (HCMS) apoptosis (30). In the present study, NRF1 was markedly reduced in the articular cartilage of 
OA model rats, as well as in LPS-stimulated chondrocytes, and NRF1 knockdown significantly enhanced chondrocyte apoptosis in vitro. In consideration of the role of NRF1 in apoptosis, the present study investigated the expression of NRF1, and found that NRF1 was markedly reduced in the articular cartilage of OA model rats, as well as in LPS-stimulated chondrocytes. Therefore, the present results suggested that NRF1 may be a potential regulator in chondrocytes apoptosis. The present study constructed a siRNA-NRF1, found that NRF1 knockdown significantly enhanced chondrocyte apoptosis in vitro.

miRNA expression is closely associated with apoptotic death in different pathological progressions (31). miRNA-410-3p protected hypoxia-induced proliferation suppression and apoptosis stimulation in cardiomyocytes via targeting TRAF5 (32). In addition, miR-425 suppressed cell apoptosis by targeting AMPH-1 in non-small-cell lung cancer (33). miRNAs are important factors for regulating the RNA network, with individual miRNAs potentially being pro-apoptotic or anti-apoptotic in certain situations (34). The present study aimed to reveal miRNAs capable of binding to NRF1 and to assess their regulatory importance in the context of chondrocyte apoptosis in OA. In the present study, NRF1 was identified as a target of miR-363-3p in chondrocytes. Previous research has demonstrated that miR-363-3p is an important regulator of apoptosis. miR-363 inhibition in cardiomyocytes reduced the incidence of hypoxia-induced apoptotic death through enhancing Notch signaling (20). The upregulation of miR-363-3p accelerated apoptosis in laryngeal cancer cells by targeting induced myeloid leukemia cell differentiation protein 1 (35). In addition, when expressed ectopically in HT29 and HCT116 cells, miR-363-3p promoted apoptosis (36). Thus, the present study hypothesized that miR-363 may represent a novel therapeutic target in OA.

IL-1 $\beta$, IL-6 and TNF- $\alpha$ are crucial cytokines in vivo (37-39). A previous study reported that the initiation of apoptosis was due to the activation of the homologous IL-1 $\beta$-converting enzyme protease family; it converts the newly synthesized precursor of IL- $1 \beta$ into active IL- $1 \beta$, and results in the breakdown of matrix proteins that maintain the structure and function of cells, in addition to mediating apoptosis by activating other members of the family (37). IL-6 affects the growth, differentiation and gene expression of numerous cell types, and its disrupted expression is closely related apoptosis (38). TNF- $\alpha$ has a potent apoptotic effect, and its apoptotic signal is mediated by the TNF receptor 1 (TNFR1) (39). In the present study, overexpression of miR-363-3p significantly increased TNF- $\alpha$, IL-1 $\beta$ and IL- 6 expression levels in synovial fluid. The present results suggested that inhibition of miR-363-3p exerted its protective effects against OA by suppressing proinflammatory cytokines induced apoptosis.

$\mathrm{Bcl}-2$ is an oncogene identified by Tsujimoto et al (40) in follicular lymphoma. Bcl-2 family members are divided into two categories according to their function; members that promote cell apoptosis, such as Bax; and members that inhibit cellular apoptosis, such as Bcl-2, and the balance between Bax/Bcl-2 controls the onset of apoptosis (41). Caspase-3 exists in the cytoplasm in an inactive format, where it coordinates signals from multiple apoptotic pathways, and activation of caspase-3 can initiate the degradation phase of apoptosis $(42,43)$. p53 is a recognized apoptotic gene vital to apoptosis, and it contributes by both inhibiting cellular proliferation and inducing cellular apoptosis $(44,45)$. In vitro, the present results suggested that miR-363-3p overexpression enhanced p53 and cleaved caspase-3 expression, inhibited Bcl-2 expression, which indicated the aggravated chondrocyte apoptosis, whereas inhibiting miR-363-3p resulted reduced apoptosis. In addition, elevated miR-363-3p expression increased apoptosis by downregulating NRF1 gene expression in vivo. In order to further investigate the role of miR-363-3p in OA chondrocyte apoptosis, supplementary experiments were conducted. The present results suggested that downregulation of miR-363-3p led to an increased expression of Bcl-2, decreased expression of p53 and cleaved caspase-3, which indicated that inhibition of miR-363-3p reduced chondrocyte apoptosis. In conclusion, the present results suggested that miR-363-3p inhibited NRF1, and this was linked with OA-associated chondrocyte apoptosis.

\section{Acknowledgements}

Not applicable.

\section{Funding}

The present study was supported by The National Natural Science Foundation of China (grant no. 81901413).

\section{Availability of data and materials}

All data generated and analyzed during the present study are included in this article.

\section{Authors' contributions}

MZ, ZQW and BJL performed the experiments, contributed to data analysis and wrote the manuscript. MZ, FYS and AZC analyzed the data. MZG conceptualized the study design, and contributed to data analysis and experimental materials. All authors read and approved the final manuscript.

\section{Ethics approval and consent to participate}

The Animal Care and Use Committee of Shandong University (Shandong, China) approved all animal studies, which were conducted in a manner consistent with the National Institutes of Health Guide for the Care and Use of Laboratory Animals.

\section{Patient consent for publication}

Not applicable.

\section{Competing interests}

The authors declare that they have no competing interests.

\section{References}

1. Xie F, Kovic B, Jin X, He X, Wang M and Silvestre C: Economic and humanistic burden of osteoarthritis: A systematic review of large sample studies. Pharmacoeconomics 34: 1087-1100, 2016.

2. Gu YT, Chen J, Meng ZL, Ge WY, Bian YY, Cheng SW, Xing CK, Yao JL, Fu J and Peng L: Research progress on osteoarthritis treatment mechanisms. Biomed Pharmacother 93: 1246-1252, 2017.

3. Barnett R: Osteoarthritis. Lancet 391: 1985, 2018. 
4. Ribitsch I, Mayer RL, Egerbacher M, Gabner S, Kańduła MM, Rosser J, Haltmayer E, Auer U, Gültekin S, Huber J, et al: Fetal articular cartilage regeneration versus adult fibrocartilaginous repair: Secretome proteomics unravels molecular mechanisms in an ovine model. Dis Model Mech 11: dmm033092, 2018.

5. Kim JH, Jeon J, Shin M, Won Y, Lee M, Kwak JS, Lee G, Rhee J, Ryu JH, Chun CH and Chun JS: Regulation of the catabolic cascade in osteoarthritis by the zinc-ZIP8-MTF1 axis. Cell 156 730-743, 2014

6. Ma F, Li G, Yu Y, Xu J and Wu X: MiR-33b-3p promotes chondrocyte proliferation and inhibits chondrocyte apoptosis and cartilage ECM degradation by targeting DNMT3A in osteoarthritis. Biochem Biophys Res Commun 519: 430-437, 2019.

7. Evans MJ and Scarpulla RC: NRF-1: A trans-activator of nuclear-encoded respiratory genes in animal cells. Genes Dev 4: 1023-1034, 1990.

8. Scarpulla RC: Nuclear respiratory factors and the pathways of nuclear-mitochondrial interaction. Trends Cardiovasc Med 6 : 39-45, 1996.

9. Zhang Y and Manning BD: mTORC1 signaling activates NRF1 to increase cellular proteasome levels. Cell Cycle 14: 2011-2017, 2015.

10. Zhang GM, Deng MT, Lei ZH, Wan YJ, Nie HT, Wang ZY, Fan YX, Wang F and Zhang YL: Effects of NRF1 on steroidogenesis and apoptosis in goat luteinized granulosa cells Reproduction 154: 111-122, 2017.

11. Niu N, Li Z, Zhu M, Sun H, Yang J, Xu S, Zhao W and Song R: Effects of nuclear respiratory factorl on apoptosis and mitochondrial dysfunction induced by cobalt chloride in $\mathrm{H} 9 \mathrm{C} 2$ cells. Mol Med Rep 19: 2153-2163, 2019.

12. Zhang $\mathrm{H}$, Wu J, Keller JM, Yeung K, Keller ET and Fu Z: Transcriptional regulation of RKIP expression by androgen in prostate cells. Cell Physiol Biochem 30: 1340-1350, 2012.

13. Berezikov E, Guryev V, van de Belt J, Wienholds E, Plasterk RH and Cuppen E: Phylogenetic shadowing and computational identification of human microRNA genes. Cell 120: 21-24, 2005.

14. Zhang S, Chen L, Jung EJ and Calin GA: Targeting microRNAs with small molecules: From dream to reality. Clin Pharmacol Ther 87: 754-758, 2010.

15. Bao J, Li D, Wang L, Wu J, Hu Y, Wang Z, Chen Y, Cao X, Jiang C, Yan W and Xu C: MicroRNA-449 and microRNA-34b/c function redundantly in murine testes by targeting E2F transcription factor-retinoblastoma protein (E2F-pRb) pathway. J Biol Chem 287: 21686-21698, 2012.

16. Zhao Z, Dai XS, Wang ZY, Bao ZQ and Guan JZ: MicroRNA-26a reduces synovial inflammation and cartilage injury in osteoarthritis of knee joints through impairing the NF- $\kappa \mathrm{B}$ signaling pathway. Biosci Rep 39: BSR 20182025, 2019.

17. Ma Y, Wu Y, Chen J, Huang K, Ji B, Chen Z, Wang Q, Ma J, Shen $S$ and Zhang J: miR-10a-5p promotes chondrocyte apoptosis in osteoarthritis by targeting HOXA1. Mol Ther Nucleic Acids 14: 398-409, 2019.

18. Ding Y, Wang L, Zhao Q, Wu Z and Kong L: MicroRNA93 inhibits chondrocyte apoptosis and inflammation in osteoarthritis by targeting the TLR4/NF- $\kappa \mathrm{B}$ signaling pathway. Int J Mol Med 43: 779-790, 2019.

19. Witkos TM, Koscianska E and Krzyzosiak WJ: Practical aspects of microRNA target prediction. Curr Mol Med 11: 93-109, 2011.

20. Meng X, Ji Y, Wan Z, Zhao B, Feng C, Zhao J, Li H and Song Y: Inhibition of miR-363 protects cardiomyocytes against hypoxia-induced apoptosis through regulation of Notch signaling. Biomed Pharmacother 90: 509-516, 2017.

21. Wenzhao L, Jiangdong N, Deye S, Muliang D, Junjie W, Xianzhe $\mathrm{H}$, Mingming Y and Jun $\mathrm{H}$ : Dually regulatory roles of HMGB1 in inflammatory reaction of chondrocyte cells and mice. Cell Cycle 18: 2268-2280, 2019.

22. Ni Z, Kuang L, Chen H, Xie Y, Zhang B, Ouyang J, Wu J, Zhou S, Chen L, Su N, et al: The exosome-like vesicles from osteoarthritic chondrocyte enhanced mature IL-1 $\beta$ production of macrophages and aggravated synovitis in osteoarthritis. Cell Death Dis 10: $522,2019$.

23. Livak KJ and Schmittgen TD: Analysis of relative gene expression data using real-time quantitative PCR and the 2(-Delta Delta C(T)) method. Methods 25: 402-408, 2001.

24. Mankin HJ: Biochemical and metabolic abnormalities in osteoarthritic human cartilage. Fed Proc 32: 1478-1480, 1973.

25. Pope JE, McCrea K, Stevens A and Ouimet JM: The relationship between NSAID use and osteoarthritis (OA) severity in patients with hip and knee OA: Results of a case control study of NSAID use comparing those requiring hip and knee replacements to those in whom surgery was not recommended. Med Sci Monit 14: CR604-CR610, 2008.
26. Onuora S: Osteoarthritis: Cartilage matrix stiffness regulates chondrocyte metabolism and OA pathogenesis. Nat Rev Rheumatol 11: 504, 2015.

27. Yudoh K and Karasawa R: Statin prevents chondrocyte aging and degeneration of articular cartilage in osteoarthritis (OA). Aging (Albany NY) 2: 990-998, 2010.

28. Héraud F, Héraud A and Harmand MF: Apoptosis in normal and osteoarthritic human articular cartilage. Ann Rheum Dis 59: 959-965, 2000.

29. Sant KE, Hansen JM, Williams LM, Tran NL, Goldstone JV, Stegeman JJ, Hahn ME and Timme-Laragy A: The role of NRF1 and Nrf2 in the regulation of glutathione and redox dynamics in the developing zebrafish embryo. Redox Biol 13: 207-218, 2017.

30. Zhang J, Gu JY, Chen ZS, Xing KC and Sun B: Astragalus polysaccharide suppresses palmitate-induced apoptosis in human cardiac myocytes: The role of NRF1 and antioxidant response. Int J Clin Exp Pathol 8: 2515-2524, 2015.

31. Su Z, Yang Z, Xu Y, Chen Y and Yu Q: MicroRNAs in apoptosis, autophagy and necroptosis. Oncotarget 6: 8474-8490, 2015.

32. Teng YL, Ren F, Xu H and Song HJ: Overexpression of miRNA410-3p protects hypoxia-induced cardiomyocyte injury via targeting TRAF5. Eur Rev Med Pharmacol Sci 23: 9050-9057, 2019.

33. Jiang L, Ge $\mathrm{W}$ and Geng J: miR-425 regulates cell proliferation, migration and apoptosis by targeting AMPH-1 in non-small-cell lung cancer. Pathol Res Pract 215: 152705, 2019.

34. Wu G, Tan J, Li J, Sun X, Du L and Tao S: miRNA-145-5p induces apoptosis after ischemia-reperfusion by targeting dual specificity phosphatase 6. J Cell Physiol: Mar 18, 2019 doi: $10.1002 /$ jcp.28291 (Epub ahead of print).

35. Feng WT, Yao R, Xu LJ, Zhong XM, Liu H, Sun Y and Zhou LL: Effect of miR-363 on the proliferation, invasion and apoptosis of laryngeal cancer by targeting Mcl-1. Eur Rev Med Pharmacol Sci 22: 4564-4572, 2018.

36. Dong J, Geng J and Tan W: MiR-363-3p suppresses tumor growth and metastasis of colorectal cancer via targeting SphK2. Biomed Pharmacother 105: 922-931, 2018.

37. Lee KH and Kang TB: The molecular links between cell death and inflammasome. Cells 8: E1057, 2019.

38. Zhu Y, Saito K, Murakami Y, Asano M, Iwakura Y and Seishima M: Early increase in mRNA levels of pro-inflammatory cytokines and their interactions in the mouse hippocampus after transient global ischemia. Neurosci Lett 393: 122-126, 2006.

39. Liu T, Bao YH, Wang Y and Jiang JY: The role of necroptosis in neurosurgical diseases. Braz J Med Biol Res 48: 292-298, 2015.

40. Tsujimoto Y, Cossman J, Jaffe E and Croce CM: Involvement of the bcl-2 gene in human follicular lymphoma. Science 228: 1440-1443, 1985.

41. Liu C, Shi Z, Fan L, Zhang C, Wang K and Wang B: Resveratrol improves neuron protection and functional recovery in rat model of spinal cord injury. Brain Res 1374: 100-109, 2011.

42. Clark WM, Rinker LG, Lessov NS, Hazel K, Hill JK, Stenzel-Poore $M$ and Eckenstein F: Lack of interleukin-6 expression is not protective against focal central nervous system ischemia. Stroke 31: 1715-1720, 2000

43. Glushakova OY, Glushakov AA, Wijesinghe DS, Valadka AB, Hayes RL and Glushakov AV: Prospective clinical biomarkers of caspase-mediated apoptosis associated with neuronal and neurovascular damage following stroke and other severe brain injuries: Implications for chronic neurodegeneration. Brain Circ 3: 87-108, 2017.

44. Fridman JS and Lowe SW: Control of apoptosis by p53 Oncogene 22: 9030-9040, 2003.

45. Lu Q, Rau TF, Harris V, Johnsonm M, Poulsen DJ and Black SM: Increased p38 mitogen-activated protein kinase signaling is involved in the oxidative stress associated with oxygen and glucose deprivation in neonatal hippocampal slice cultures. Eur J Neurosci 34: 1093-1101, 2011.

his work is licensed under a Creative Commons Attribution-NonCommercial-NoDerivatives 4.0 International (CC BY-NC-ND 4.0) License. 